\title{
FUZZY LOGIC, DEDUCTIVE RULES OF INFRENCE AND LINGUISTIC REASONING ON KNOWLEDGE BASE
}

\author{
NGUYÊN CÁT HỒ \\ Institute of Information Technology
}

\begin{abstract}
Summary. In this paper we introduce a notion of knowledge base consisting of statments with truth degree in which every statment may have several truth degrees. A set of rules of inference handling this kind of statments, a deductive reasoning method based on these rules will be considered. The consistency of the knowledge base will be also investigated.
\end{abstract}

\section{INTRODUCTION}

In [2] and [7] we gave an algebraic approach to fuzzy logic by axiomatizing linguistic domains of linguistic variables, especially, of the linguistic truth variable, based on partially ordered linguistic meaning structure of vague concepts. This means that linguistic domains can be considered as algebras in the category of universal algebras and called Extended Hedge Algebras, briefly, EHGs. They are so rish algebras, that the operations corresponding to logical connectives are definable in these stuctures. In other words, the considered domains of linguistic values are closed w.r.t. logical connectives. This suggests us to investigate in this paper fuzzy logic based on EHGs and establish a deductive system for linguistic reasoning, analogously as in classical and non-classical logics.

There will be some foundations for building up this deductive system for linguistic reasoning. First, it is able to introduce a fuzzy logic, which is axiomatized, based on properties of EHAs - a class of universal algebras. It may be important in constructing inference rules for linguistic reasoning. Remember that every deductive system in classical or non-classical logics always determines an algebra in a certain class of universal algebras (see [1], [4] and [15]). Second, analogously as in the case of classical or non-classical logics (see [1], [10], [14]), many inference rules for linguistic reasoning can be justified by truth valuations, based on the stuctures of domains of the linguistic truth variable. The other rules will be seen emperically reasonable and it will be shown that the results obtained by this way are not worse than that based on fuzzy sets reasoning.

It is wirth to mention that our rules of inference allow to manipulate immediately vague concepts and the results are given in a form of natural language. 
Finally, we could introduce a notion of derivation from a given set of fuzzy sentences, called assumptions, which can be considered as a knowledge base. Then we have a method in linguistic reasoning to deduce step by step a conclusion formulated in natural language. For example, in the sequel we shall see that from the following information: 'the more healthy a peson is, the more efficiently he or she does his or her job" is of truth degree 'rather true' and 'Robert is very healthy' is of truth degree 'possibly true', we can derive by means of our infegrence rules a conclusion that 'Robert does his job very efficiently' is of truth degree 'rather possibly true'.

It will be seen that this method seems to be rather simple and applicable.

The paper is continued that published in [6] and some results of the paper can be find also in it without proof.

\section{FUZZY PROPOSITIONS AND LINGUISTIC VALUATION}

It is known that people are thinking and reasoning by languages, whose fundametal elements are sentences normally containing vague concepts. Sentences in human reasoning have implicitly or explicitly, a truth degree which is often expressed also by vague concepts such as "true", "false", "possibly true" and so on. For example, the sentence 'John is high' in a certain context may be assigned to a truth degree "very true".

- So, in many situations we are dealing with sentences containing value concepts associated with a linguistic truth degree. This leads to a consideration of the set of fuzzy sentences and a notion of linguistic truth valuation, which assigns to certain fuzzy sentences linguistic truth values.

A simple form of fuzzy sentences are those which can be called fuzzy predicates. Examples of such sentences are the following: 'Robert is very old', 'The gas gauge reads normal', 'Part $X$ of the engine depends on part $Y$ strongly' and the motor turns well'. Because we want to deal with vague concepts, they will be divoiced from the other and therefore fuzzy predicates will be devided into two parts. The first one is those which build the main meaning of such sentences, i.e. it consists of all words excepts vague concepts occuring in the sentences. They can be considered as ordinary predicates and called the substance part. Examples are '(the age of) Robert is in the first sentence above, 'The gas guage reads' in the second, 'Part $X$ of the engine depends on part $Y$ ' in the third and 'The motor turns'.

The second part of fuzzy predicate consists of the remaining words - the vague concepts. It is called the value part. In the above sentences they are 'very old', 'normal', 
'strongly' and 'well'. It can be seen that the substance part determines the possible vague concepts which are interpreted as its values. Therefore, the substance part plays a role similarly as that of linguistic variables. Hence, fuzzy predicates can be denoted by a pair $(p, u)$, where $\mathrm{p}$ is a predicate of $\mathrm{n}$ arguments and $\mathrm{u}$ is a vague concept. By this notation, the above examples can be expressed by ( $A G E$ (Robert), veryold), (READ (gasgauge), normally), (DEPE$N D(X, Y)$, strongly)and(TURN(motor), well), respectively. In the sequel, we shall see that this formalization will be very convernient for manipulation of vague concepts in our method.

The simple fuzzy sentences of the forms considered above are called elementary fuzzy propositions.

The composed fuzzy propositions are formed from elementary ones by means of logical connectives such as 'and', 'or', 'if... then...' and 'not', which are denoted correspondingly by,$\rightarrow$ and $\neg$ and called conjunction, disjunction, implication and negation. An example for composed fuzzy propositions is 'if a person is old then he or she does not run quickly' and this sentence can be expessed by $(A G E(x)$, old $) \rightarrow \neg(R U N(x)$, quickly $)$.

Now we shall give a formalization to describe the set of fuzzy propositions. -

(i) Linguistic domains as sets of terms: Every predicate $p$ will be associated with a linguistic domain $D(p)$, which is embeded in an extended hedge algebra (EHA). For example, predicate $A G E(x)$ is associated with an EHA generated by the primary terms 'old' and 'young', predicate $R E A D(x)$ is associated with the one generated by 'normally' and 'unnormally' and so on.

In the paper, we shall consider only linguistic domains generated by two generators and, then, they are symmetrical EHGs (see [CW91]), in which there is operation -, where for every element $u,-u$ is the contradictory element of $u$. So, every linguistic domain $D(p)$ can be represented by $(D(p), C, H(p),-)$, where $H(p)$ is the set of hedges and $C$ is the set of the primary vague concepts associated with $p$ and regarded as the zero-argument operations.

Let us consider a set $S Y M$ of symbols which consists of the elements of $C, H(p)$ and of the symbol -. We denote by $T S$ the set of all strings of symbols in $S Y M$.

The set of terms corresponding to domain $D(p)$ is defined as the smallest subset $T E R(p)$ of $T S$ satisfying the following conditions:

(t1) $C \subseteq T E R(p)$;

(t2) For any $h$ in $H(p)$, and $u$ in $T E R(p), h u$ is in $T E R(p)$;

(t3) For any $u$ in $T E R(p),-u$ is in $T E R(p)$.

(ii) The set $F P$ of fuzzy Propositions: 
Let us denote by $V$ ar and $C o n$ the sets of individuals and constants, respectively and considere a finite set $P R$ of predicates. Elements in $P R$ will be denoted by small letters $p, q, r, \ldots$ with indeces if necessary. To every element $p$ of $P$ it will be associated with linguistic domain $D(p)$. The logical connectives are $\wedge, \vee, \rightarrow$, and $\neg$. The auxiliary symbols are brackets (, ), and comma ",". Denote by $T F$ the set of all strings of elements in $S Y M$ and in $P R$ and of logical connectives.

$F P$ is the smallest subset of $T F$ satisfying the following conditions:

(p1) For every $p$ in $P R$ and $u$ in $T E R(p),(p, u)$ is in $F P$, i.e. $F P$ consists of all elementary fuzzy propositions. For convenience, for $(p, u) \in F P$ and a hedge $h$, some times we shall write $h(p, u)$ instead of $(p, h u)$.

(p2) For $P, Q$ in $F P, P \vee Q$ is in $F P$;

(p3) For $P, Q$ in $F P, P \wedge Q$ is in $F P$;

(p4) For $P, Q$ in $F P, P \rightarrow Q$ is in $F P$;

(p5) For $P$ in $F P, \neg P$ is in $F P$.

Now we difine a linguistic truth valuation $v$.

Let us considered a symmetrical hedge algebra $T=(T, C, H,-, \cup, \cap, \Rightarrow, \leq)$ of the linguistic truth variable, where $C=\{$ True, False $\}$.

We assume that all sets $H(p)$ with $p$ in $P R$ and $H$ are isomorphic in the category of the partially ordered sets and hence, they are assumed to be the same.

By linguistic truth valuation, we mean an arbitrary partial mapping $v: F P \rightarrow T$ satisfying the following conditions:

- (v1) For any elementary fuzzy proposition $P$ in $F P$, if $P$ has no individual variables then $v(P)$ is defined;

(v2) For $F=h P, v(P)$ is defined and equals to $\sigma$ ha, where $a \in C$, iff $v(h P)$ is defined and equals to $\sigma \mathrm{a}$.

(v3) $v(\neg P)=-v(P)$, if $v(P)$ is defined;

(v4) $v(P \vee Q)=v(P) \cup v(Q)$, if $v(P)$ and $v(Q)$ are defined;

(v5) $v(P \wedge Q)=v(P) \cap v(Q)$, if $v(P)$ and $v(Q)$ are defined;

(v6) $v(P \rightarrow Q)=v(P) \Rightarrow v(Q)$, if $v(P)$ and $v(Q)$ are defined;

\section{ASSERTIONS AND KNOWLEDGE}

The main purpose of the paper is to model human reasoning based on its knowledge and the author thinks that every person's knowledge contains sentences with a certain truth degree, which normally is also a linguistic concept. They will be called in our study 
assertions. So, every assertion is a pair $(F, t)$, where $F$ is a fuzzy proposition and $t$ is a linguistic truth value being interpreted as a truth degree. The human being has an ability to deduce some new assertions from a given set of other ones. We shall introduce an approach to a human reasoning process that are based on rules of inference allowing to handle directly linguistic concepts.

In the incomplete information phenomena it can be seen that (i) one has no definitive way to deduce exactly one conclusion in fuzzy phenomena, (ii) There exist many basises to justify the reason why one assigns one or other truth degree to a given statment and, hence, every statment may have several truth degrees. This suggests us to define a formal knowledge as a set of assertions as follows.

Let $F P$ be the set of fuzzy proposition considered in the previous section. Let $\sim>$ be an additional logical connective which is called proportional implication, the semantics of which will be examined later. The set $A$ of assertions is the least set of strings of symbols satisfying the following conditions

(A1) For every $P \in F P$ and $t \in T,(P, t) \in \mathcal{A}$

(A2) For any $P, Q \in F P, t \in T,(P \sim Q, t) \in \mathcal{A}$.

The assertion $(P \sim P, t)$ can be considered as an abreviation of the set assertions $\{\sigma P \rightarrow \sigma Q: \sigma$ is an abitrang string of hedges $\}$.

Let $K$ be a subset of $A$, we denote by $F P(K)$ the set $\{P:(P, t) \in K\}$ and $T_{K}(P)=$ $\{t:(P, t) \in K\}$.

A subset $K$ of $A$ is said to be a formal knowledge provided, for every $P \in(F P(K)$, either $T_{K}(P)>W$ or $T_{K}(P)<W$, where $W$ is the neutral in $T$.

Interpretation of formal knowledge: The main aim of reasoning methods is to model human reasoning. Thus, one of the main subjects of the study is human being knowledge.

A human being knowledge may be of one person or of a collective of persons such as a group of experts. As we discussed previously, a human knowledge is a set of pair $(P, t)$ where $P$ is a fact in the real world $R W$ and $t$ is a belief degree which is represented in the study by a linguistic truth value. We shall take human knowledges as models of our formal knowledge.

To explain this, let us consider $Q$ as a set of objects in the real world and. I is an interpretation which

(i0) interpret every variable as an object in $\Omega$.

(i1) interpret every constants as a certain object in $\Omega$.

(i2) interpret individual variables as variables over $\Omega$.

(i3) interpret every predicate $p$ as a relation $R p$ over $\Omega$. 
(i4) interpret primary terms and hedges of a predicate $p$ as the primary terms and hedges of associated with relation $R p$.

(i5) interpret connetives $\neg, \vee, \wedge,-->$ correspondingly as $-, \cup, \cap$ and $\Rightarrow$,

A model for a formal knowledge is a structure $M=\left(\Omega,\left\{R_{p}, H\left(R_{p}\right): p \in P\right\}, \tau_{0}, V a l, T\right)$, where:

$\Omega$ is a set of objects, $\tau_{0}$ is a mapping which assigns to every constan $\mathrm{c}$ an object $O_{c}$ in $\Omega R_{p}$ is a relation over $\Omega . H\left(R_{p}\right)$ is the set of hedges of $R_{p}$ which is assumed to be is ormorphic to $H(T) . V a l$ is a set of partial valuations, which for every $R_{p}$ and for some objects $O 1, \ldots, O n$ and a primary term $u$ of $P$, assign to $\left(P_{I}(O 1, \ldots, O n), u\right)$ a truth value in $T$.

For a formula $F, V C(F)$ denotes the set of all individual variables and individual constants occuring in $F$, and $F$ denote the expression obtained from $F$ by replacing every predicate $p$ by $P_{I}$. Let us denote by $T$, an extension of $\tau_{0}$ over the set of all individual variables. As a convention, for $F=P o Q$ if $v(P)$ and $v(Q)$ are defined then $v(F)$ is defined and $v(F)=v(P) \circ v(Q)$ and $v(F i(\ldots)) \in T_{K}(F)$ means that if $v(F i(\ldots))$ is defined then it belongs to $T_{K}(F)$. For the formular of the form $F=P \sim Q$, where $P, Q \in F P$, the equality $v(P \sim Q)=t$ means that, for every string of hedges a, $v(a P \rightarrow a Q)=t$.

A formal knowledge $K$ of $\left(F, T_{K}(F)\right)$ is said to be satisfied in $M$ if for every $v \in V$ of $M, v$ is defined on $F P(K)$ and $v(P) \in F(F)$ for every $F \in F P(K)$. Two formulars $P$ and $Q$ in $F P$ are said to be equivalent provided for every evaluation $v$, if $v(P)$ and $v(Q)$ are defined then $v(P)=v(Q)$. In this case we shall write $P \equiv Q$. It can easily be seen that the following statements hold:

Theorem 3.1. For any formulars $F, P, Q$ and any predicate $p$, hedge $h$, the following statement hold.

(i) $\neg(P, u) \equiv(p,-u)$ and $(p,-h u) \equiv(p, h-u)$ :

(ii) $P \equiv P$ and $\neg P \equiv P$ :

(iii) $P \wedge Q \equiv Q \wedge P$ and $P \vee Q \equiv Q \vee P$;

(iv) $F \wedge(P \wedge Q) \equiv(F \wedge P) \wedge Q$ and $F \vee(P \wedge Q) \equiv(F \vee P) \vee Q$;

(v) $F \wedge F \equiv F$ and $F \wedge F \equiv F$;

(vi) $F \vee(F \wedge P) \equiv F$ and $F \wedge(F \vee P) \equiv F$;

(vii) $F \vee(P \wedge Q) \equiv(F \vee P) \wedge(F \vee Q)$ and

$F \wedge(P \vee Q) \equiv(F \wedge P) \wedge(F Q)$;

(viii) $\neg(F \wedge P) \neg F \neg P$ and $\neg(F \vee P) \equiv \neg F \wedge \neg P$;

(ix) $F \rightarrow->P \neg F \vee P$.

(x) If $P \equiv Q$ and $F(P)$ means $F$ contains $P$ as its subformular, then $F(P) \equiv F(Q / P)$, where 
$F(Q, P)$ denotes the formula obtained from $F$ by replacing $P$ with $Q$.

\section{RULES OF INFERENCE IN LINGUISTICREASONING}

In this section we shall establish some rules for linguistic reasoning, with which we can manipulate vague concepts in an easy way in comparison with other methods (see $[12],[13],[15]$ and $[16])$.

Rules are schema allowing to deduce a conclusion from a given set of assertions.

Formaly, a rule of inference is a scheme of the following form:

$$
\frac{\left(P_{1}, t_{1}\right), \ldots,\left(P_{n}, t_{n}\right)}{\left(Q_{1}, s_{1}\right), \ldots,\left(Q_{m}, s_{m}\right)}
$$

where $\left(P_{i}, t_{i}\right), t_{i}>W, i=1, \ldots, n$ are called antecedents or assumptions and $\left(Q_{j}, s_{j}\right), \dot{s}_{j}>$ $W, j=1, \ldots, m$, are called.conclusions. A rule (RUL) is said to be sound provided for every valuation $v$, if $\left(P_{i}\right)$ is defined and $v\left(P_{i}\right)=t_{i}, i=1, \ldots, n$, then $v\left(Q_{j}\right)$ is defined and $v\left(Q_{j}\right)=s_{j}$, for $j=1, \ldots, m$.

\subsection{Rule for hedge transfer for simple fuzzy propositions.}

In reasoning some time we need to change an assertion to anather one, which in a definitive sense is equivalent to the former one. E.g. we want to change the assertions ('Mary is attractiove' very True), ('John is More or less young', possibly True) and ('John is very strong', More or Less False) into the form for example ('John is strong', t), for a suitable linguistic truth value $t$. The following rule of inference describes how we can realize this and determine the linguistic truth value $t$.

$$
\begin{aligned}
& \frac{((P, h u), s T)}{((P, u), s h T)} \\
& \frac{((P, u), s h T)}{((P, h u), s T)}
\end{aligned}
$$

where $\mathrm{T}$ is a primary term of the linguistic truth variable, i.e. $\mathrm{T}$ is either 'True" or 'False', $\mathrm{s}$ is a string of hedges and $\mathrm{h}$ is an arbitrary hedge.

By these rules, the above examples of assertions can be trasfered into the following ones: 'Mary is very attractive' is 'True', 'John is strong' is 'More or Less Very False' or 'John is more or less very strong' is 'False', 'John is young' is 'Possibly More or Less True' or 'John is possibly more or less young' is 'True'. more or less young' is 'True'. 
At first glance, it can be seen that the rules (RT) may be reasonable, intuitively. However, someone may doubt its reliability. Therefore, we want to show here that reasoning based on these rules is not worse than that based on fuzzy sets theory (see [8], [9], $[15]$ and [16]).

As we know, a rule which is analogous to (RT) in approximate reasoning based on fuzzy set theory is the following:

$$
\therefore \quad \frac{\text { 'u is } A^{\prime} \text { is } t}{u \text { is } B} \text {, }
$$

where $A$ is a fuzzy set, $t$ is a fuzzy set of linguistic truth variable and $B$ is the fuzzy set computed by $B=$ to $A$.

For uniformity, the proposition ' $u$ is $B$ ' in the conclusion of Rule (ST) needs be understood as the assertion ' $u$ is B' is 'True', where True is assumed to be the label of the fuzzy set $\mu_{\text {True }}(v)=$, for $v \in[0,1]$. Now, we give an example to show that when vague concepts can be represented by fuzzy sets, Rules (RT) have the same effect as Rule (ST). Let us consider the following proposition 'Robert is young' is 'Very True'. It can be verified that $\mu_{V \text { eryTrue }} O \mu_{\text {young }}(u)=\mu_{\text {True }} o \mu_{V \text { eryYoung }}(u)$, the fuzzy set on the left side, on account of the rule (ST), represents the sentence " 'Robert is young' is 'Very True'" and the fuzzy set on the right side, again by the rule (ST), represents the sentence "'Robert is very young' is 'True"', which is just what is deduced from the given sentence by the rule (RT). However, (RT) is more advantageous in manipulating vagueness, especially, for the case where vague concepts are difficult to be represented by fuzzy sets, e.g. vague concepts of beauty as 'attractive',.... For example, from the given assertion 'Mary is very attractive' is 'Possibly True' it can be deduced that 'Mary is possibly very attractive' is 'True' or 'Mary is attractive' is 'Possibly Very True'. It is worth to mention that by Rule (ST) we are not able to infer these assertions.

By the definition of the valuation, we have

Proposition 3.1. Rules (RT1) and (RT2) are sound.

Proof. By (V2) of the definition of valuation.

4.2. Rules of hedge trasfer for implication.

First, we introduce a notation. Let $v$ be a given linguistic truth valuation. Denote by $\operatorname{dom}(v)$ the set of all fuzzy propositions $P$, where $v(P)$ is defined. We shall define expression of the form $h P$ with respect to $v$ and $h$ is a hedge, by induction as follows: 
For the primary propositions $P=P(x, h u)$ we shall write $P=h P(x, u)$. In this case we have always the fact that if $v P(x, h u)=s T$, where $s$ is a string of hedges and $T \in C$ is a truth constant, then $v(P(x, u))=s h T$, by the definition of valuation.

For $P=\neg h Q$, if $v(\neg h Q)=\sigma T$ implies that $v(\neg Q)=\sigma h T$, then we shall write $P=h \neg Q$;

For $P=h Q o h Q^{\prime}$, where o is a two argument logical connective, if $v(P)=\sigma T$ implies that $\left(Q \circ Q^{\prime}\right)=\sigma h T$, then we shall write $P=h\left(Q \circ Q^{\prime}\right)$.

It is worth to mention that the notation $h P$ means that the hedge $h$ can be trasfered as in the case of Rules (RT1) and (RT2). As a convention, if $h P$ occurs in an expression, we mean $h$ can be transfered.

Note that $\mathrm{h}$ can not be always transfered. But we have the following statements. To formulate these, we need a notion. $P$ and $Q$ are said to be consistent w.r.t. $v$ if $v(P)>W$ and $v(Q)>W$, and inconsistent if one of $v(P)$ and $v(Q)$ is greater than $W$ and the other is less than $W$.

From now on, when we write $h P$, it means that $h$ can be transfered w.r.t. the set of valuations $v$, which are definitively determined.

Lemma 4.2. Let $v$ be given. Then

(i) $\neg h P=h \neg P$;

(ii) $h P \vee h Q=h(P \vee Q)$, if $P$ and $Q$ are inconsistent w.r.t. v;

(iii) $h P \wedge h Q=h(P \wedge Q)$, if $P$ and $Q$ are inconsistent w.r.t. v;

(iv) $h P \rightarrow h Q=h(P \rightarrow Q)$, if $P$ and $Q$ are consistent w.r.t. v.

Proof: (i) Let $v(\neg h P)=\sigma T$. By the property of $v, v(h P)=-\sigma T=\sigma-T$, which implies, by definition of the formular $h P$, that $v(P)=\sigma h-T=-\sigma h T$ (see [7]). Again by property of $v, v(\neg P)=\sigma h T$. Thus, the validity of (i) has been proved.

(ii) Suppose that $v(h P \vee h Q)=\sigma T, v(h P)=\sigma_{1} T_{1}$ and $v(h Q)=\sigma_{2} T_{2}$, where $\sigma, \sigma_{i}$ ar strings of hedges and $T, T_{i}$ are primary terms 'True' or 'False'. Since $P$ and $Q$ are inconsistent w.r.t. $v$, it follows that either $T_{1}=$ 'True' and $T_{2}=$ 'False' or T1 $=$ 'False' and $T_{2}=$ 'True'. Assuming that $T_{1}=$ 'False', we have $v(h P) \leq v(h Q)$ and so $v(h P \vee h Q)=v(h P) \vee v(h Q)=\sigma_{2} T_{2}=\sigma T$. The last equality shows that $\sigma_{2}=\sigma$ and $T_{2}=T$. By the definition, $v(h P)=\sigma_{1} T_{1}$ and $v(h Q)=\sigma_{2} T_{2}$ imply that $v(P)=\sigma_{1} h T_{1}$ and $v(Q)=$ $\sigma_{2} h T_{2}$. Since $T_{1}=$ 'False' and $T_{2}=$ 'True', it is obvious that $\sigma_{1} h T_{1} \leq \sigma_{2} h T_{2}$ and hence $v(P \vee Q)=v(P) \vee v(Q)=v(Q)=\sigma_{2} h T_{2}=\sigma h T$, which proves the validity of (ii).

(iii) is pvoved by duality and (iv) follows from (ii), since $v(h P \rightarrow h Q)=v(\neg h P) \cup v(h Q)$.

Remark: By the definition, the notation $h P$ means that $h$ may be transfered. On account of Lemma 3.2 , it is obvious that the condition for hedge transfer is determined 
by the truth values of $P$ and $Q$, but does not depend on hedges. Therefore, instead of writting $h P$ to denote the fact that $h$ can be transfered, we can also say that $P$ allows to transfer hedges. In addition, by the definition of the formular of the form $h F$, it forllows that Rule (RT) for primary propositions is also correct for the proposition allowing to transfer hedges. Hence, Rule (RT) can be formulated as follows:

$$
\begin{aligned}
\frac{(h P, s T)}{(P, s h T)}, \\
\frac{(P, s h T)}{(h P, s T)} .
\end{aligned}
$$

4.3. Rules of hedge transfer for implication, Modus ponens and Modus tolens

Rules of hedge transfer:

$$
\begin{gathered}
\frac{(h P \rightarrow h Q, \sigma \text { True }),(h P, s \text { True })}{(P \rightarrow Q, \sigma h T \text { rue })} \\
\frac{(P \rightarrow Q, \sigma h T r u e),(P, \text { shTrue })}{(h P \rightarrow h Q, \sigma T r u e)}
\end{gathered}
$$

where $\sigma$ and a are strings of hedges.

Rule of Modus ponens:

$$
\frac{(P \rightarrow Q, \sigma \text { True }),(P, \text { True })}{(Q, \sigma \text { rue })}
$$

Rule of modus tolens:

$$
\frac{(P \rightarrow Q, a T r u e),(\neg Q, \text { True })}{(\neg P, \sigma \text { True })}
$$

The reasonableness of these rules is based on the following statement:

Proposition 4.3. Rules (RTI1), (RTI2), (RMP) and (RMT) are sound.

The proof of this proposition is based on Lemma 3.2 with a notice that $P$ and $Q$ or $h P$ and $h Q$ are consistent (w.r.t. the valuations to be considered).

\subsection{Rule of proportion implication}

Now we introduce a special rule to interpret a feature of linguistic implication that may be adopted in several phenomena. To justify this rule we study some examples.

Let us consider the following assertion: 'if something is rare then it is expensive' is 'rather true'. We can observe that there exists a proportion between the meanings of the vague concepts in the two parts of the sentence 'if..., then...'. This means that 'if what is 
rarer, then it is more expensive' and 'if what is less rare, then it is less expensive'. These sentences can be understood to have the same truth degree as the given one does. This leads to the following assertions:'If a thing is very rare, then it is very expensive' is 'rather true' or 'If a thing is more or less rare then it is more or less expensive' is 'rather true'.

We may give many other examples which describe the analogous situation, e.g. 'If somebody is healthy, then he is able to work hardly'.

It is wirth to quote here, as examples, statements about experiences of some asian nationalities: 'If the farmer has enough warter resourse and enough fertilizer, then the rice will be good'; 'If the parents take care their children carefully, then they will be a good boy'; 'If a boy learns actively, then his results should be good' and so on. It can be seen that these statements have the same property described above, i.e. the premises and the conclusions of these 'if..., then...' sentences involve a proportion meaning relationships. Therefore, we shall call this kind of sentences proportion implications. This implication can be viewed as a family of the implications of the form $\alpha P \rightarrow \alpha Q$, where $\alpha$ is an arbitrary string of hedges.

Let proportion implication be denoted by $\sim>$. The above observation can be formalized by the following rules of inference.

Rule of proportion impplication is, then, stated as follows:

$$
\frac{(P(x, u) \sim>Q(x, v), \sigma \text { True })}{(\alpha P(x, u) \rightarrow \alpha Q(x, v), \sigma \text { True })}
$$

where $\alpha$ and $\sigma$ are strings of hedges, $P$ and $Q$ are formulars allowing to transfer hedges.

Rule of substitution of individual constant for individual variable

$$
\frac{P(x, u)}{P(a, u)}
$$

where $\mathrm{a}$ is an individual constant For convernience in the sequel, we introduce the following rule. Rule of equivalence:

$$
\frac{P \leftrightarrow Q,(F(P), \alpha T)}{(F(Q), \alpha T)}
$$

where $F(X)$ is a formular containing $X$ as a subformular.

\section{LINGUISTIC REASONING}

The main problem of human reasoning is that: given a set $K$ of assertions, which facts can be deduced from $K$ ? This problem often arises in expert systems or in decision 
FUZzY LOGIC, DEDUCTIVE RULES OF INFERENCE AND LINGUISTIC REASONING ...

support systems, where knowledge is represented by rules. We assume that assertions in $K$ have truth degrees expressed by linguistic concepts of 'Truth', i.e. of the form sTrue, and they will be called assumptions.

Now we define a notion of a derivation from $K$ by means of the rules of inference considered in Section 4.

A derivation from $K$ is a finite sequence of assertions $(P 1, t 1), \ldots,\left(P_{n}, t n\right)$ such that, for every $i=1, \ldots, n$, either $(P i, t i)$ belongs to $K$ or $(P i, t i)$ is obtained from $(P 1, t 1), \ldots,(P i-$ $1, t i-1)$ by one of the rules (RT), (RTI1), (RTI2), (RMP), (RMT), (RPI), (RSUB) and (RE), i.e. $(P i, t i)$ is a conclusion of an inference rule, whose antecedents are some of the assertions $(P 1, t 1), \ldots,(P i-1, t i-1)$ In this case, $(P n, t n)$ is called a consequence of $K$ and denoted by the notation $\mathrm{K} \vdash(P n, t n)$.

Given $K$, we shall denote by $C(K)$ the set of all consequences of $K$, i.e. $C(K)=$ $\{(P, t): K \vdash(P, t)\}$.

$K$ is said to be consistent if there is a valuation $v: F P \rightarrow\{0,1\}$ such that for every $(P, t) \in K, v(P)=1$ iff $t>W$. Such a valuation $v$ is called two-valued $K$-valuation.

We shall show that the definition of consistency of $K$ is natural. Since we allow every formular may have several linguistic truth values, but they all are either greater than $W$ or less than $W$, both assertions $(P, t)$ and $(P, s)$, where $t \neq s$ and $t>W$ and $s>W$, may be contained in the same knowledge $K$. However if, for example $t>W$ and $s<W$, we get an absurd. Now, we establish an equivalence relation $\sim$ on the set $F P$ by induction on the length of formulas, which satisfies the following:

(FR1) $p(x, h u) \sim P(x, u)$ for any predicate $p$, hedge $h$ and any term $u$.

(FR2) if $P \sim P^{\prime}$ then $\neg P \sim \neg P^{\prime}$

(FR3) if $F \sim F^{\prime}, Q \sim Q^{\prime}$ then $P o Q \sim P^{\prime} \circ Q^{\prime}$, for any two argument connectives $o \in\{\vee, \wedge, \rightarrow, \sim>\}$. Especcially, $P \rightarrow Q \sim P^{\prime} \rightarrow Q^{\prime}$.

Denote by $|P| \sim$ the equivalence class containing $P$ and by $F P_{\sim}$ the set $\left\{|P|_{\sim}: P \in\right.$ $F P\}$. Moreover, we have $|P|_{\sim}=|\neg P|_{\sim},|P|_{\sim}|Q|_{\sim}=|P Q| \sim$ and $|P|_{\sim} \rightarrow|Q|_{\sim}=|P \rightarrow Q|_{\sim}$ or $|P|_{\sim} \rightarrow|Q|_{\sim}=|P \sim>Q|_{\sim}$. Notice that for every $P \in F P,|P|$ is a formular of a first order predicate language. So, we can regard $F P / \sim$ as the set of open formulas of a language of a classical first order logic

For $t, s \in T$, we shall write $t \approx s$ if either $t=s$ or $t>W$ and $s>W$ or $t<W$ and $s<W$. Evidently, $\approx$ is an equivalence relation and the set of equivalence classes over $T$

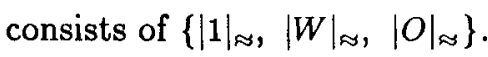

For any two assertions $A=(P, t)$ and $A^{\prime}=\left(P^{\prime}, t^{\prime}\right) . A$ and $A^{\prime}$ are said to be equivalent if $P \sim P^{\prime}$ and $t \approx t^{\prime}$ and we shall write $A \equiv A^{\prime}$. 
The class of all assertions which are equivalent to $A$ is denoted by $|A|$.

Given $K$, denote by $K / \equiv$ the set $\left\{|A|_{\approx}: A \in K\right\}$. Note that if $K$ is a knowledge, then $(P, t)$ and $\left(P^{\prime}, t^{\prime}\right) \in K$, implies $t \approx t^{\prime}$. Thus if $(P, t) \in K$ then $|P| \sim$ has a unique truth value $|t| \approx$, if $K$ is a kernel of a knowledge, then for $|A|_{\approx} \in K_{\curvearrowright},|A|_{\approx}=\left(|P|_{\sim},|1|_{\circledast}\right)$.

By $K / \equiv 十_{c}|A|$ we understand that $|A|_{\approx}$ can be deduced from $K / \equiv$ by means of rules of inference (RSUB) and (RMP).

Theorem 5.1. $K \vdash A$ implies that $K / \equiv \vdash|A|$ and $K$ is consistent iff $K / \equiv$ is consistent.

Proof Let $A_{1}, . ., A_{n}$ be a derivation from $K$ for $A$.

If $A_{j}$ derived from $A_{i}, i<j$, by one of the rules (RT1) or (RT2), (RT1') and (RT2'), then it is obvious from the definition of $\equiv$ that $\left|A_{j}\right|=\left|A_{i}\right|$.

If $A_{j}$ is deduced from $A_{i}, A_{l}, \mathrm{i}$ and $l<j$, by rules (RTI1) or (RTI2) and assume that $\mathrm{Ai}$ is the first antecedent of (RTI1) or (RTI2) then it can be seen that $\left|A_{j}\right|=\left|A_{i}\right|$, since

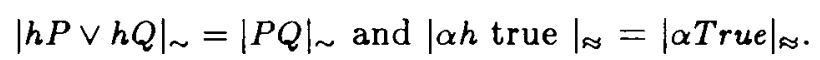

If $A_{j}$ is deduced from $A_{i}$, where $i<j$, by rules (RPT) then evidently $\left|\hat{A_{j}}\right|=$ $\left(|\alpha P(x, u) \rightarrow \alpha Q(x, u)|,|1|_{\circledast}\right)=\left|A_{i}\right|$.

If $A_{j}$ is deduced from $A_{i}$ and $A_{l}$, where $i, l<j$, by rules (RMP) or (RMT), thèn it can be seen that $\left|A_{j}\right|$ can be deduced from $\left|A_{i}\right|,\left|A_{j}\right|$ by rules modus ponens and modus to lens.

Since $P \leftrightarrow Q,|P|$ and $|Q|$ are equivalent in the sense of classical firt ordered logic. It follows that $\left(|P|_{\sim} \rightarrow|Q|_{\sim}\right) \wedge\left(|Q|_{\sim} \rightarrow|P|_{\sim}\right)$ and hence if $A_{i}$ is derived from $A_{j}, A_{l}$ by rule (RF) then it can be verified that $\left|A_{i}\right|,\left|A_{l}\right| \vdash_{c}\left|A_{j}\right|$.

Let $v$ be a two-valued valuation. Recall that 0 and 1 are fixed points, i.e. $h 0=0$ and $h 1=1$ for all $h \in H$, therefore from the definition of a valuation, if $p(x, h u) \sim p(x, u)$ then $v(p(x, h u))=v(p(x, u))$. By induction on the length of formulas, it can be seen that if $P \sim P^{\prime}$ and $Q \sim Q^{\prime}$ then $v(P \circ Q)=v\left(P^{\prime} \circ Q^{\prime}\right)=v\left(P^{\prime}\right) \circ v\left(Q^{\prime}\right)$ for any two-argument connectives o and $v(P)=v\left(\neg P^{\prime}\right)=-v\left(P^{\prime}\right)$. Thus, it follows that every two-valued valuation $v$ induced a valuation $v_{\sim}$ over $F P / \sim$ and, hence if $K$ is consistent then $K / \equiv$ is also consistent in the sense of open theory of a first order logic.

Conversly, if $v_{\sim}$ is a valuation of $K / \equiv$ such that for $\left(|P|_{\sim},||_{*}^{*}\right) \in K / \equiv, v^{\sim}(|P| \sim)=$ $|t|_{\approx}$, then $v_{\sim}$ induces a two-valued valuation $v$ for $K$. Therefore, if $K / \equiv$ is consistent then so is $K$.

It implies that, if $K$ is consistent, then both $K \vdash(p, t)$ and $K \vdash(\neg p, s)$, where $t, s>W$, do not hold simultaneously.

Theorem 5.2. Let $K$ be a formal knowledge. Then 
(i) If $K \vdash(P, t)$ then $t>W$

(ii) If $K$ is consistent then so is $C(K)$.

Proof. The statemient (i) can be proved by the observation that for every $A=(Q, s) \in K$, we have $\operatorname{Truth}(A)=s>W$ and, it can be cheked that all rules of inference preserve the property that if $\operatorname{Truth}\left(A_{i}\right)>W$ for all premises of a rule of inference, then $\operatorname{Truth}(B)>W$, where $B$ is the conclusion of this rule of inference.

(ii) Suppose that $K$ is consistent. As it has been proved above, $K / \equiv$ is consistent, too, and if $(P, t) \in C(K)$ then $K /_{\equiv \vdash_{c}}\left(|P|_{\sim}, 1\right)$, i.e. $|P|_{\sim}$ belongs to an open theory $T$ with axioms to be the formulas in $K / \equiv$. Since $K / \equiv$ is consistent so is $T$ and hence there is a model for $T$, i.e. there exists a valuation $v^{\sim}$ such that $v^{\sim}\left(|P|_{\sim}\right)=1$ for all $|P|_{\sim} \in T$ (see [RS68]). As shown above, $v^{\sim}$ induces a two-valued valuation $v$ for $C(K)$, i.e. $C(K)$ is consistent.

Now, we give some examples to illustrate our method.

Example 1. Let us receive the following information: (i) "The more healthy a man is, the more efficient he does his job is 'Rather True" and

(ii) "Robert is possibly healthy' is 'Very True".

The question is what conclusions can we deduce from these informations. In order to answer this question we build a derivation as follows: Let us denote the sentence ' $x$ is healthy' by $\mathrm{p}(\mathrm{x}$,healthy) and ' $\mathrm{x}$ does his or her job efficiently' by $\mathrm{q}(\mathrm{x}$,efficiently).

(1) ( $p$ (Robert,possibly healthy), Very True), (by the assumpton);

(2) ( $\mathrm{p}$ (Robert,very possibly healthy),True), (byRule(RT));

(3) (p(x,healthy) > q(x,efficiently), Rather True), (by the assumption);

(4) (very possibly $p$ (Robert,healthy) $\rightarrow$ very possibly q(Robert,efficiently), Rather True), (by Rule (RPI1));

(5) (very possibly q(Robert,efficiently), Rather True),(by Rule (RMP));

(6) ( $q($ Robert,very possibly efficiently),Rather True),(by Rule (RE));

(7) (q(Robert,possibly efficiently), Rather Very True),(by Rule (RT));

(8) (q(Robert,efficiently),Rather Very Possibly True),(by Rule (RT)).

Example 2. In this example we shall show that although hedges in concaternetion seem to be difficult to understand in practice, but it is important in our method to represent the degree of the reliability of fuzzy sentences.E.g. suppose that we receive, in addition, another more information: (iii) "Roll is healthy' is 'more possibly True". By a similar derivation as in Example 1 above, we obtain:

(9) (q(Roll,efficiently),Rather More Possibly True). 
From (8) and (9), it follows that Robert does his job better than Roll, since 'Rather More Possibly True' is less than 'Rather Very Possibly True'.

Example 3. We consider now a more complicated example. Les us receive the following informations:

(i) 'If a student work hardly and his university is authorized, then he will be a good employee' is 'true';

(ii) 'The University where Robert studies is very authorized' is 'possibly true';

(iii) 'Robert is studying very hard'.

The question is what conclusions can be deduced from these informations? Denote by $p(a, h a r d)$ the sentence ' $x$ works hard', by $q(U(x)$, authorized)- the sentence 'the university of $x$ is authorized' and by $r(x, g o o d)$ the sentence ' $x$ is a good employee'. Then we can construct the following derivation:

1) ( $q(U($ Robert ), very authorized), Possibly True), (by assumption);

2) ( $q(\mathrm{U}($ Robert), possibly very authorized), True), (by Rule (RT));

3) ( $\mathrm{p}(\mathrm{U}$ (Robert), rather hard), True),(by the assumption);

4) $(\mathrm{p}(\mathrm{x}$, hardly $) \wedge \mathrm{q}(\mathrm{U}(\mathrm{x})$, authorized $) \rightarrow \mathrm{r}(\mathrm{x}$,good $)$, True $)$, (by the assumption);

5) $(\mathrm{q}(\mathrm{U}(\mathrm{x})$, authorized $) \sim>(\mathrm{p}(\mathrm{x}$, hardly $) \rightarrow \mathrm{r}(\mathrm{x}$, good $))$, True $)$, (by Rule (RE))

6) (Possibly very $q(\mathrm{U}($ Robert), autherized $\rightarrow$ Possibly Very (p(Robert), rather hard) $\rightarrow \mathrm{r}$ (Robert,good)), True), (5) Rule (RPI),(RPI) and rule (RSUB)

7) (Possibly Very (p(Robert,rather hard) $\rightarrow \mathrm{r}$ (Robert,good)), True), (2),6) and Rule (RMP));

8) $((p$ (Robert,rather hard $) \rightarrow r($ Robert,good),Possibly Very True), (7) and Rule(RT'1))

9) $(p$ (Robert,rather hard $) \rightarrow p($ Robert,rather good),Poss Very True), (8) and Rule(RPI)

10) ( r(Robert,rather good), Poss Very True), ((3), 9) ,Rule(RMP));

11) r(Robert,Poss Very Rather good), True), (10), Rule(RT));

It is worth to mention that $Q \rightarrow(P \rightarrow Q)$ and $P \rightarrow(Q \rightarrow R)$ are requivalent formular, however if we use the latter instead of the first one occuring in 5 ) then we shall obtain (r(Robert,Rather Poss Very good),True), the truth degree of which is different from that of the assertion in 11). This shows that, the ordering of subformular $p(x$, hardly) and $q(U(x)$, authorized $)$ is important, although they are equivalent in our semantics by Rule (RE). The author thinks that the ordering of these subformulars in descending of their importance degree, i.e. subformular $\mathrm{p}(\mathrm{x}$,hardly) has a importance degrees greater than that of $q(U(x)$, authorized $)$, is appropriate to our intuition. Thus, we consider the assertion 11 ) is better than the above one.

Conclusion: In this paper we have tried to give an approach to human reasoning 
FUZZY LOGIC, DEDUCTIVE RULES OF INFERENCE AND LINGUistic REASONING ...

by means of deductive systems. we consider the knowledge of every person as a set of sentences associated with certain. linguistic belief degrees and believe that one can derive a conclusion from its knowledge in a similar way as that based on classical logic. In order to realise this, we have first constructed mathematical structure of truth linguistic value, the ordering relation of which can be determined by their natural meaning.

The research was supported in part by the Vietnam National Fundamental on Natural Sciences.

\section{REFERENCES}

1. N. Cat Ho, Generalized Post algebras and their application to some infinitary ma ny-valued logic, Dissertationes Mathematicae CVII (1973),pp 1-76.

2. N. Cat Ho, Fuzziness in the structure of linguistic truth values: a foundation for development of fuzzy reasoning, Proc. of Int. Symp. on Multiple-Valued logic, Boston University, Boston, Massachusetts, May 26-28, IEEE Computer Society Press,1987,pp. 325-335.

3. N. Cat Ho and H. Rasiowa, Semi-Post algebras, Studia Logica, XLVI,2 (1987), pp. 149-160.

4. N. Cat Ho and H. Rasiowa, Subalgebras and homomorphisms of semi-Post algebras, - ibid. , pp. 161-175.

5. N. Cat Ho and H. Rasiowa, Plain semi-Post algebras and their representability. Studia Logica Vol.48,4(1989),01-22.

6. Nguyen Cat Ho, Linguistic-valued logic and a deductive Method in linguistic reasoning, Proceeding of the Fifth IFSA World Congress, Seoul, Korea, July 4-9, 1993.

7. N. Cat Ho and W. Wechler, Hedge algebras: an algebraic approach to structures of sets of linguistic domains of linguistic truth variable, Fuzzy Sets and Systems, Vol. 35,3, pp.281-293(1990)

8. Satoru Fukami et al., On fuzzy reasoning, System. Computer. Control, 9(1978),pp. 44-53.

9. R. Giles, Lukasiewicz logic and fuzzy set theory, Int. J. Man-Machine Studies 8(1976), pp. 313-327.

10. H. Rasiowa, An algebraic approach to non-classical logic, North-Holland, London/New York, 1974.

11. H. Rasiowa and R. Sikorski, the Mathematics of Metamathematics, second ed. revised, Warszawa, 1968. 
12. P. Smets and p. Magnez, Implication in fuzzy logic,(1985), submitted for publication.

13. Yahachiro Tsukamoto, An approach to fuzzy reasoning method, in M.M. Gupta, R.k. Ragade, R.R. Yager (eds.), Advances in Fuzzy Set Theory and Applications, North-Holland Publishing Company(1979),pp. 137-149.

14. L.A. Zadeh, Fuzzy-set-theoretic interpretation of linguistic hedges, Journal of Cyber. 2(1972),pp. 4-34.

15. L.A. Zadeh, The concept of linguistic variable and its application to approximate reasoning (I), (II), Information. Science 8(1975),pp. 199-249; 8(1975),pp. 301-357.

16. L.A. Zadeh, Fuzzy logic and approximate reasoning, Synthese 30(1975),pp. 407-428. 